\title{
Successive approximation algorithm for beam-position-monitor-based LHC collimator alignment
}

\author{
Gianluca Valentino, ${ }^{1,2, *}$ Andriy A. Nosych, ${ }^{1,3,4}$ Roderik Bruce, ${ }^{1}$ Marek Gasior, ${ }^{1}$ \\ Daniele Mirarchi, ${ }^{1}$ Stefano Redaelli, ${ }^{1}$ Belen Salvachua, ${ }^{1}$ and Daniel Wollmann ${ }^{1}$ \\ ${ }^{1}$ CERN, CH-1211 Geneva 23, Switzerland \\ ${ }^{2}$ University of Malta, Msida MSD2080, Malta \\ ${ }^{3}$ The University of Liverpool, Liverpool L69 7ZX, United Kingdom \\ ${ }^{4}$ The Cockcroft Institute, Daresbury WA4 4AD, United Kingdom
}

(Received 4 November 2013; published 24 February 2014)

\begin{abstract}
Collimators with embedded beam position monitor (BPM) button electrodes will be installed in the Large Hadron Collider (LHC) during the current long shutdown period. For the subsequent operation, BPMs will allow the collimator jaws to be kept centered around the beam orbit. In this manner, a better beam cleaning efficiency and machine protection can be provided at unprecedented higher beam energies and intensities. A collimator alignment algorithm is proposed to center the jaws automatically around the beam. The algorithm is based on successive approximation and takes into account a correction of the nonlinear BPM sensitivity to beam displacement and an asymmetry of the electronic channels processing the BPM electrode signals. A software implementation was tested with a prototype collimator in the Super Proton Synchrotron. This paper presents results of the tests along with some considerations for eventual operation in the LHC.
\end{abstract}

DOI: 10.1103/PhysRevSTAB.17.021005

PACS numbers: 41.85.Si, 29.20.dk, 29.50.+v

\section{INTRODUCTION}

The Large Hadron Collider (LHC) is a high-energy circular collider located at CERN. It is designed to accelerate $3.23 \times 10^{14}$ protons in two counterrotating beams to achieve collisions in the experimental insertions at a center-of-mass energy of $14 \mathrm{TeV}$ [1]. The LHC is composed of eight arcs and eight straight sections, called insertion regions (IRs). A beam cleaning or collimation system is in place to intercept potentially dangerous beam halo particles, therefore preventing their deposition in the superconducting magnets or other sensitive areas of the machine [2]. A total of 108 collimators are installed, mainly in IR3 and IR7 for momentum and betatron offset cleaning, respectively.

An LHC collimator is $1 \mathrm{~m}$ long and is made up of two movable copper blocks (jaws) with a graphite, tungsten, or copper layer on each jaw surface facing the beam, as shown in Fig. 1. The extremities (corners) of each jaw can be moved independently by dedicated stepping motors in $5 \mu \mathrm{m}$ steps. Each jaw is classified as left or right, depending on its location with respect to the incoming beam. The collimators have different transverse rotations, with the rotation angle defined as the clockwise angle with respect

*gianluca.valentino@cern.ch

Published by the American Physical Society under the terms of the Creative Commons Attribution 3.0 License. Further distribution of this work must maintain attribution to the author(s) and the published article's title, journal citation, and DOI. to the negative horizontal axis. A collimator with a zero angle thus collimates the beam in the horizontal plane, while a collimator with a $90^{\circ}$ angle collimates in the vertical plane. The upstream jaw corners are those that see the oncoming beam, while the downstream corners see the beam exiting the collimator.

During LHC operation, the collimator jaws are opened to various gaps, centered around the actual beam orbit and forming a four-stage hierarchy to clean with the maximum efficiency $[3,4]$. The primary collimators are positioned closest to the beam, followed by the secondary collimators (TCSG), tertiary collimators (TCT), and absorbers. The correct positions are achieved only if the beam axis and beam size at the collimator locations are known precisely. A beam-based alignment procedure was adopted at the LHC during the 2010-2013 runs [5,6], in which these parameters are determined by moving the jaws inwards on either side of the beam, until a beam loss spike is detected separately for the left and right jaws.

The alignment procedure was recently automated, using feedback from the beam loss monitors (BLMs) $[7,8]$ and automatic classification of the resultant loss signal spikes [9] to reduce the alignment time of all collimators from around $30 \mathrm{hr}$ in 2010 to less than $4 \mathrm{hr}$ in 2012 . However, the limitations of the BLM-based alignment technique have been reached. The collimator jaws can be moved inwards with a finite movement rate, and the feedback algorithm needs to wait until the beam losses decay back to a steady state after each loss spike before resuming the alignment. The decay time varies from 3 to up to $20 \mathrm{~s}$, and is inversely 


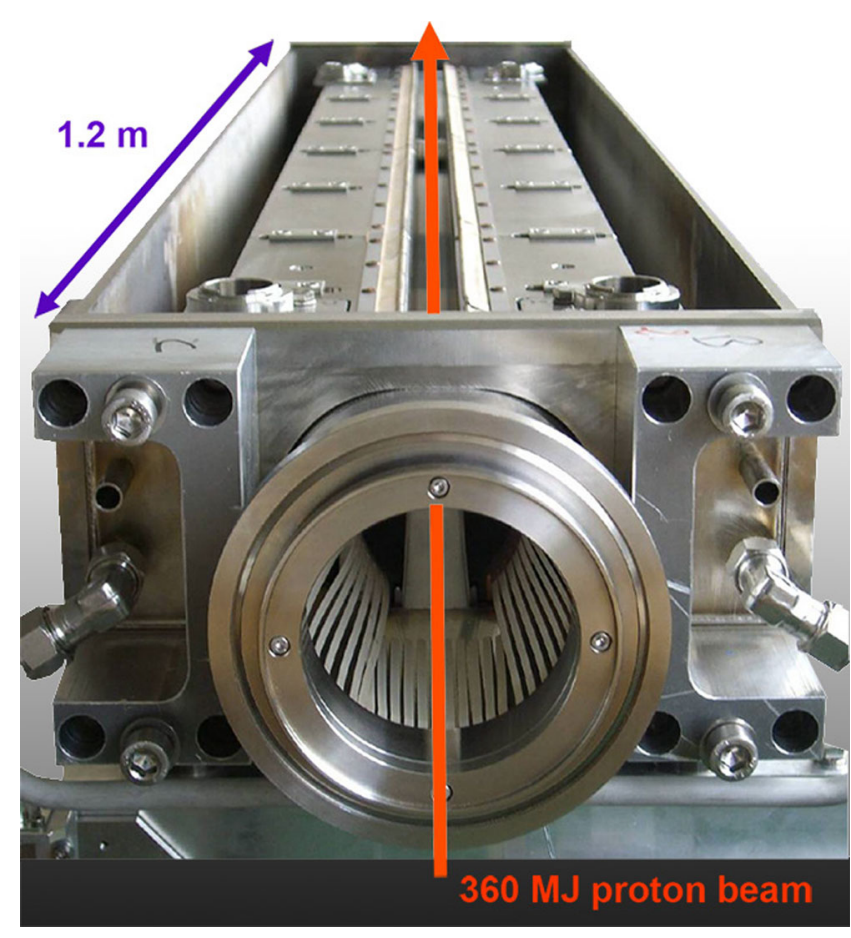

FIG. 1. Photograph of the LHC collimator as viewed from one end. The jaws are enclosed in a 1.2-m-long casing.

proportional to the cut in units of $\sigma$ made by the collimator jaw. In addition, BLM-based alignments require dedicated low-intensity fills, which reduce the beam time available for physics operation. The lack of frequent alignments means that large margins need to be placed on the hierarchy settings to cater for possible beam orbit drifts over weeks or months of operation. This contributes to a high $\beta^{*}$ (the $\beta$ function in the interaction points), which reduces the luminosity reach of the LHC.

A faster alignment can be achieved if BPMs are embedded in the collimator jaws [10]. This new design envisages the installation of two BPM buttons per jaw, with one button embedded in each jaw corner. A model of a jaw is shown in Fig. 2. The button electrodes in the jaw corners are installed in a flat surface retracted by $10.6 \mathrm{~mm}$ from the active surface of the jaw, and are hence protected from possible direct beam impacts. In standard operation, BPMs

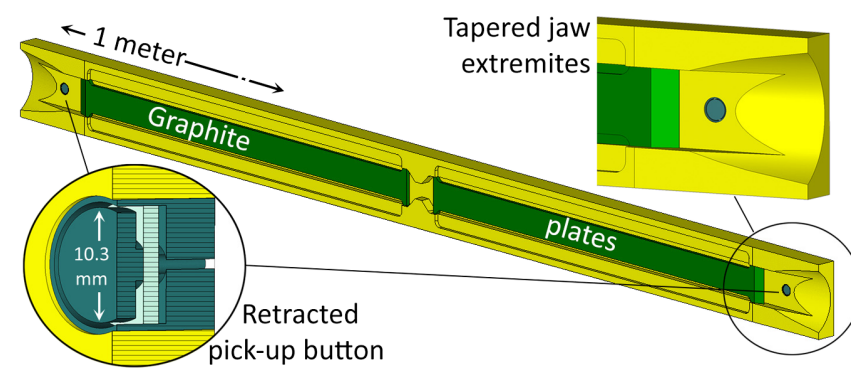

FIG. 2. Model of a jaw with embedded BPM button electrodes as installed in the prototype collimator in the SPS. will help to eliminate all orbit-related settings errors at the collimator locations. They will provide online monitoring of the beam position, including the possibility of placing interlocks on the orbit measurements. The use of embedded BPM collimators in operation can help to improve the $\beta^{*}$ reach by about $15 \%$ [11].

A prototype collimator equipped with embedded BPMs (see Fig. 2) was installed in the CERN Super Proton Synchrotron (SPS) in January 2010, oriented for beam cleaning in a horizontal plane. A number of beam feasibility tests were performed in 2010-2011, including verification of the alignment accuracy $[12,13]$. The successful results from the beam tests motivated the replacement of all 16 TCTs located in the experimental IRs (eight horizontal and eight vertical) and the two TCSGs in IR6 (both horizontal) with new collimators having embedded BPM buttons for LHC operation in 2015. The installed collimators will be similar to the prototype in the SPS, except that the graphite or tungsten surface will extend over the whole jaw instead of being partitioned into two plates as in Fig. 2. The next stage was to test the full data acquisition chain, correction of BPM nonlinearities, and alignment algorithm to benefit from the operational gains offered by the new hardware and reduce the current alignment time required of $1 \mathrm{hr}$ for all TCTs. The beam tests were performed with the same prototype collimator in the SPS in 2012 .

This paper is structured as follows. An overview of the BPM coordinate system is presented in Sec. II, while the BPM data acquisition is explained in Sec. III. This is followed by the various corrections applied to the measurements in Sec. IV. In Sec. V, the alignment algorithm together with beam test results are presented.

\section{BPM COORDINATE SYSTEM}

\section{A. Collimator coordinate system}

Figure 3 shows a schematic of the collimator coordinate system and several further defined notations. The prototype collimator consists of two copper jaws and a 10-mm-thick tapered graphite layer (resistivity $13 \mu \Omega \mathrm{m}$ ) on each jaw surface. The four stainless steel button electrodes of diameter $10.3 \mathrm{~mm}$ are placed at upstream and downstream jaw extremities $0.6 \mathrm{~mm}$ below the copper level, i.e., $10.6 \mathrm{~mm}$ below the graphite surface [14]. With such a setup, the total distance $B$ between opposite upstream or downstream BPM electrodes is

$$
B=J_{L}-J_{R}+2 \times 10.6=G+21.2,
$$

where $J_{L}$ and $J_{R}$ are the left and right jaw positions with respect to absolute axis, and $G$ is the distance between the graphite surfaces of the opposite jaws. We will refer to $B$ as the BPM aperture, and to $G$ as the jaw gap. Depending on the collimator type, the jaws may be positioned for LHC operation at gaps ranging from 2 to $60 \mathrm{~mm}$. 


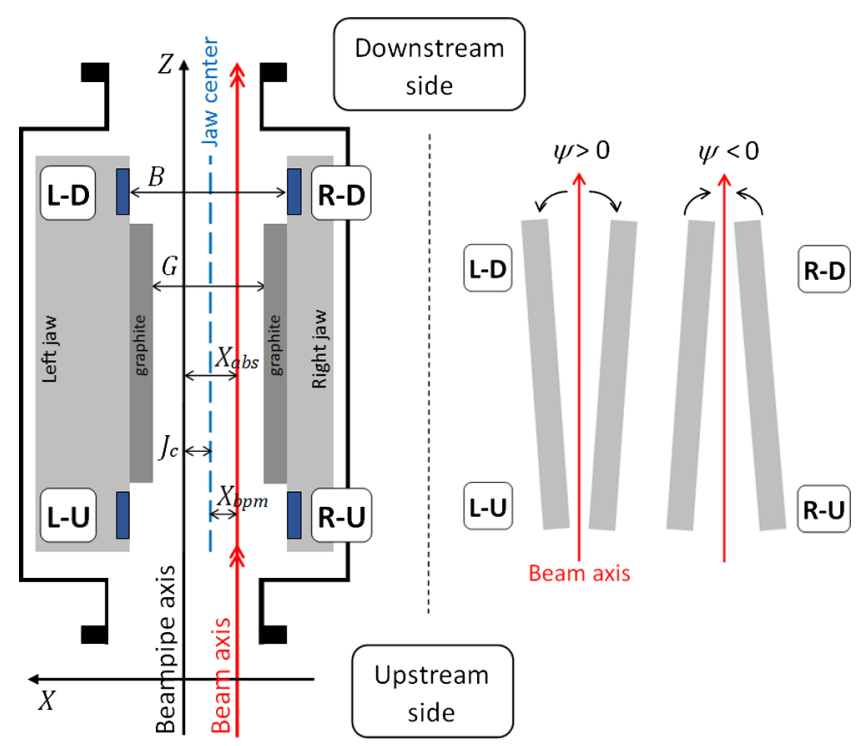

FIG. 3. The collimator coordinate system (left) and the jaw tilt angular convention (right) as viewed from above, from [13]. The four motors positioned at the edges of each jaw allow the leftupstream (LU), left-downstream (LD), right-upstream (RU), and right-downstream (RD) corners to be moved individually.

In this paper, for simplicity, the upstream and downstream jaw corners will not be treated separately as they are mechanically identical. However, at the orbit processing or jaw alignment level, all discussed calculations must be applied independently to upstream and downstream pairs of BPMs.

The absolute axis is defined as a geometrical reference axis of the beampipe, connected at both ends of the collimator. The beam traveling inside the beampipe may have an arbitrary offset from its absolute axis in the transverse plane. Since the BPMs are placed in the $X Z$ plane only, this offset is considered as horizontal and defined as beam axis or $X_{\text {abs. }}$. The collimator tank is aligned with the beampipe with an accuracy of $\sim 100 \mu \mathrm{m}$ during installation to ensure that $X_{\text {abs }}$ lies along the nominal orbit location. This unknown mechanical offset is one of the reasons why beam-based alignment is required.

In such an arrangement, a moving axis of the collimator, i.e., its midjaw position, is defined as jaw center: $J_{c}=\left(J_{L}+J_{R}\right) / 2$. Collimator BPMs measure the horizontal position of the beam as $X_{\mathrm{bpm}}$ which is always relative to the jaw center. The upstream or downstream beam axes are calculated generally as

$$
X_{\mathrm{abs}}=X_{\mathrm{bpm}}+J_{c}
$$

Hence, the jaws are precisely centered around the beam when $X_{\mathrm{bpm}}=0$ and $J_{c}=X_{\mathrm{abs}}$ for both the upstream and downstream jaw corners.

\section{B. Position measurement of an offset charge}

The beam position between two BPM electrodes can be calculated directly using a well-known linear technique. However, this method might not satisfy the precision requirements (approximately $20 \mu \mathrm{m}$ ) for operation with high energy beams in the LHC. In this paper, we will demonstrate the performance of a linear method in comparison with a developed accurate correction method that involves a $2 \mathrm{D}$ polynomial.

Consider a simple 2D approximation of a BPM arrangement, which consists of a circular beampipe and two pointlike electrodes located $180^{\circ}$ apart on a horizontal axis. The horizontal position of a charged particle, located in $(x, y)$, is denoted as $X_{\mathrm{bpm}}$ and calculated from the raw BPM signals, i.e., from the difference between the induced potentials on the left $V_{L}$ and right $V_{R}$ electrodes, normalized to the sum signals:

$$
X_{\mathrm{bpm}}=k_{x} \times X_{\text {raw }}=k_{x} \frac{V_{L}-V_{R}}{V_{L}+V_{R}} .
$$

Here, $k_{x}$ is a horizontal calibration constant that serves as a linear scaling coefficient between the relative raw readings $X_{\text {raw }}$ and $\mathrm{mm}$. $X_{\text {raw }}$ is relative to the total voltage measured by both buttons and lies in the range $[-1,1]$. Such linear scaling approach is called the difference over sum (DOS) method.

The tilt of a given jaw with respect to the beam axis (for example, the left jaw) can be estimated to first order from the upstream and downstream voltage readings:

$$
\psi_{L}^{\mathrm{tilt}}=\frac{V_{\mathrm{LU}}-V_{\mathrm{LD}}}{V_{\mathrm{LU}}+V_{\mathrm{LD}}} .
$$

The output signal level of the BPM electrode is proportional to a nonlinear sensitivity function, which is given by the mirror charges intercepted by the surface of an electrode. Hence, the scaling factor $k_{x}$ consists of a nonlinear contribution of both horizontal $(x)$ and vertical $(y)$ positions of the charge and can be approximated by the Taylor series [15]:

$$
k_{x}=\frac{B}{4}\left(1-\frac{x^{2}+y^{2}}{(B / 2)^{2}}+\left[\frac{x^{2}+y^{2}}{(B / 2)^{2}}\right]^{2}-\ldots\right)^{-1} .
$$

In some cases, when the beam does not deviate far from the beampipe center $(x, y \ll B)$, a linear relationship between beam position and pick-up signals is assumed. The scaling factor is then approximated by $B / 4$ and Eq. (3) provides an approximate position of the charge between two pairs of electrodes:

$$
X_{\mathrm{bpm}} \simeq \frac{B}{4} \frac{V_{L}-V_{R}}{V_{L}+V_{R}} .
$$

The DOS linearization is handy and quick when large beam drifts are not foreseen. With large enough drifts from 
the absolute center, the normalized DOS signal suffers from nonlinear cushion effects [15]. This nonlinearity is a purely geometrical effect of the BPM and is related to the electrostatic field distribution of the potential between BPM electrodes, electrode shape, and shape of the grounded beampipe. To compensate for this undesirable effect, the according values of $k_{x}$ depending on $x$ and $y$ must be used rather than $B / 4$. One way to handle this would be to calculate $k_{x}$ a priori as a function of the jaw gap $G$ and select it accordingly during operation. However, due to a variety of different collimators this would be a clumsy approach, involving a large look-up table or a massive storage database with frequent network access. Moreover, the variable jaw gap during operation adds another dimension to the nonlinear effect of position measurement. This renders the DOS method inefficient for online orbit calculation.

A sophisticated and accurate correction of geometrical BPM nonlinearities is essential for rapid convergence of the collimator alignment algorithm. In Sec. IV, a single 2D correction polynomial will be built that will include the scaling and correction of a collimator's nonlinear BPM readings for the whole jaw motion range and possible beam offsets within defined limits.

\section{BPM DATA ACQUISITION}

\section{A. BPM dedicated electronics}

The BPM button signals are processed by electronics based on compensated diode detectors. Two diode orbit front-end prototypes were built and tested in the laboratory and with a beam [16]. In this technique the diode detectors convert nanosecond pulses from BPM electrodes into slowly varying signals, which are low-pass filtered to $10 \mathrm{~Hz}$ and sent to a 24-bit analog-to-digital converter (ADC) with a sampling frequency of a few $\mathrm{kHz}$, averaged down and sent through an ethernet link by a simple microcontroller. This type of electronics is also intended for installation in the LHC for the standard BPMs [17]. As the jaw motion can be performed only at $2 \mathrm{~mm} / \mathrm{s}$, even readings at $10 \mathrm{~Hz}$ do not limit the overall system speed. Such slow rates allow measurements to be performed over thousands of machine revolutions, resulting in submicrometer system resolution. The absolute micrometer accuracy will be achieved by calibrating the system offset and gains with the same beam signals [18].

The performance of the diode electronics was evaluated with the SPS beam. The beam is kept circulating at a given energy (120 or $270 \mathrm{GeV})$. This mode is relevant for the conditions in which the collimators will be operated in the LHC. A submicrometer system resolution was demonstrated by comparing beam positions measured at the upstream and downstream collimator BPMs. Results from such a measurement are presented in Fig. 4, showing the upstream and downstream beam positions and tilts changes

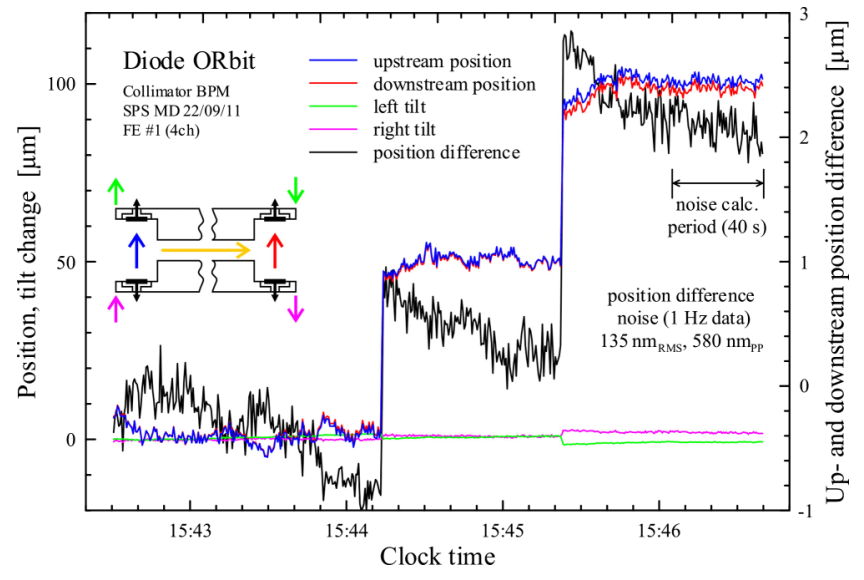

FIG. 4. Beam position and tilt measured at the upstream and downstream BPM ports and the position difference: SPS coasting beam, one bunch, $10^{11}$ protons.

when one collimator jaw was moved in two $100 \mu \mathrm{m}$ steps, resulting in a $50 \mu \mathrm{m}$ equivalent beam displacement per step. The black curve (with the scale on the right vertical axis) shows the difference of the upstream and downstream jaw positions, measured on two button electrode pairs spaced by $108 \mathrm{~cm}$, making the measurement independent of the SPS beam stability. The observed position difference steps of the order of $2 \mu \mathrm{m}$ are likely related to a small asymmetry in the motorization of each jaw end. The noise of the position difference measurement was estimated to be $140 \mathrm{~nm}(\mathrm{rms})$ for $1 \mathrm{~Hz}$ data rate. During the measurement the BPM electrode aperture $B$ was $52 \mathrm{~mm}$.

\section{B. Treatment of digital signals}

The ADC samples are averaged by a programmable factor, and are sent as user datagram protocol (UDP) network packets by a built-in microcontroller in the SPS tunnel to an intermediate server. Data from the LU, LD, RU, and RD BPM electrodes are included in the packet payload. The server forwards the packets on request to the collimator application running in the CERN Control Center or the data logging application. This is an ad hoc setup conceived to allow maximum flexibility for changing top-level control software and parameters during the beam tests.

Each packet has a 48 byte header, to which a maximum of 992 bytes of data can be appended. The individual header field names and sizes are provided in Table I. In the software application, the raw electrode data is decoded as follows. The first step is to extract the full-scale range of the ADC:

$$
R_{\mathrm{fs}}=k_{\mathrm{avg}} \times \frac{2^{24}}{2^{\left\lceil\log _{2}\left(k_{\mathrm{avg}} \times 2^{24} / 2^{32}\right)\right\rceil}},
$$

where $k_{\text {avg }}$ is the averaging factor. The data rate is determined by the averaging factor and the ADC clock frequency of $11.719 \mathrm{kHz}$ as follows: 
TABLE I. BPM UDP packet header field names and sizes.

\begin{tabular}{lcc}
\hline \hline Name & Size (bytes) & Data type \\
\hline Packet type & 10 & byte array \\
Authorization key & 8 & byte array \\
Source host mumber & 4 & int \\
Send time $(\mathrm{s})$ & 4 & unsigned long \\
Send time $(\mu \mathrm{s})$ & 4 & unsigned long \\
Acquisition timestamp & 8 & unsigned long long \\
Sequence number & 4 & unsigned long \\
ADC sample number & 2 & UINT16 \\
Averaging factor & 2 & UINT16 \\
\hline
\end{tabular}

$$
D R=\frac{f_{\mathrm{ADC}}}{k_{\mathrm{avg}}} .
$$

The electrode readings are then obtained by normalizing the received data $D$ to the ADC full scale range:

$$
V_{j}=\frac{D_{j}}{R_{\mathrm{fs}}}
$$

where $j$ denotes the jaw corner. For the beam tests with the prototype collimator, the averaging mode was used with one data sample sent per UDP at a rate of $10 \mathrm{~Hz}\left(k_{\mathrm{avg}}=1172\right)$.

\section{BPM MEASUREMENT CORRECTIONS}

\section{A. BPM nonlinearities}

A collimator computer-aided design model was used to study and characterize the BPM readouts through electromagnetic simulation by Computer Simulation Technology (CST) Particle Studio [19] in a time domain. The sensitivity of collimator BPMs to beam position was analyzed by displacing the simulated beam in the horizontal plane for a set of jaw gaps ranging from 2 to $60 \mathrm{~mm}$. In particular, for each given jaw gap, a set of beam offsets on a horizontal axis was simulated, namely, between $[-50 \%, 50 \%]$ of the jaw half gap, that is $G / 2$ (e.g., Fig. 5). The original horizontal offset of the beam with respect to the jaw center $J_{c}$ is defined as $X_{\text {beam }}$ at a corresponding step of $\Delta X$ :

$$
X_{\text {beam }, n}=J_{c}+n \times \Delta X \text {, }
$$

where $n$ is an iterator over each beam scan position. For simulation purposes, the collimator axis was aligned with the beam axis so that $J_{c}=0$ and $X_{\text {abs }}=0$. At each iteration, three values were recorded: the original beam position $X_{\text {beam }}$, the raw position $X_{\text {raw }}$ [Eq. (3)], given by peak voltages measured at the BPM ports in a time domain, and the value of $B$ which is provided by jaw positions $J_{L}$ and $J_{R}$. All simulations were performed with parallel jaws, i.e., zero tilt, resulting in the same voltages on the upstream and downstream BPM buttons on same jaw. This excluded

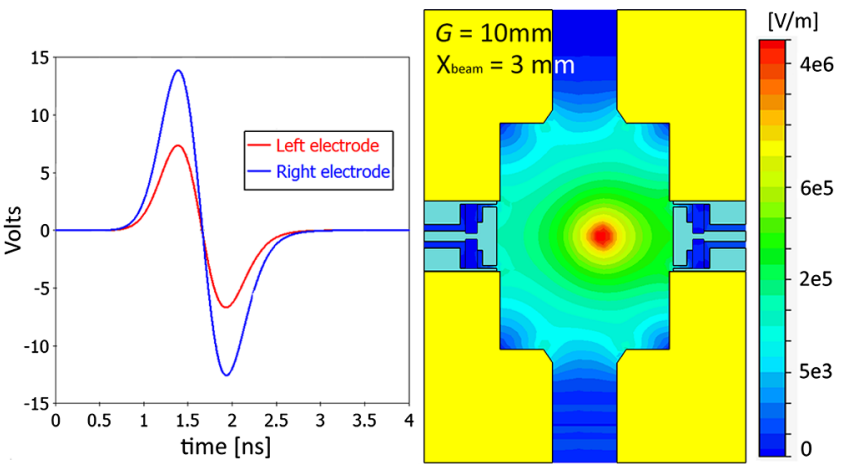

FIG. 5. Simulated time signal at 50 Ohm BPM ports (left) and snapshot of the E-field distribution of a single off-centered bunch ( $X_{\text {beam }}=30 \mathrm{~mm}, G=10 \mathrm{~mm}$ ) passing in a cross section at the upstream BPM center (right).

separate treatment of upstream and downstream BPM pairs and they were considered identical. The plots in Fig. 6 show the nonlinear relationship between $X_{\text {beam }}$ and $X_{\text {raw }}$, depending on the beam offset and jaw gap. A subset of the simulation results were successfully verified with a circulating beam in the CERN SPS ring [20].

In the perfect case, a linearized BPM reading should provide the original offset value: $X_{\mathrm{bpm}} \approx X_{\text {beam }}$. In Eq. (6) it was shown that the calibration constant amounts to $k_{x}=B / 4$ for infinitely small beam offsets. The BPM response can be then approximated to $\mathrm{mm}$ with

$$
X_{\text {bpm }}=B / 4 \times X_{\text {raw }} \approx X_{\text {beam }},
$$

and is referred to as a linearized position by the DOS method. Here, a linearity factor parameter $L_{f}$ must be introduced, which is a nonlinear conversion coefficient between the linearized and original beam positions:

$$
L_{f}=\frac{X_{\text {bpm }}}{X_{\text {beam }}} .
$$

The values of the linearity factor, calculated during horizontal beam sweep simulations as in Fig. 6, are shown

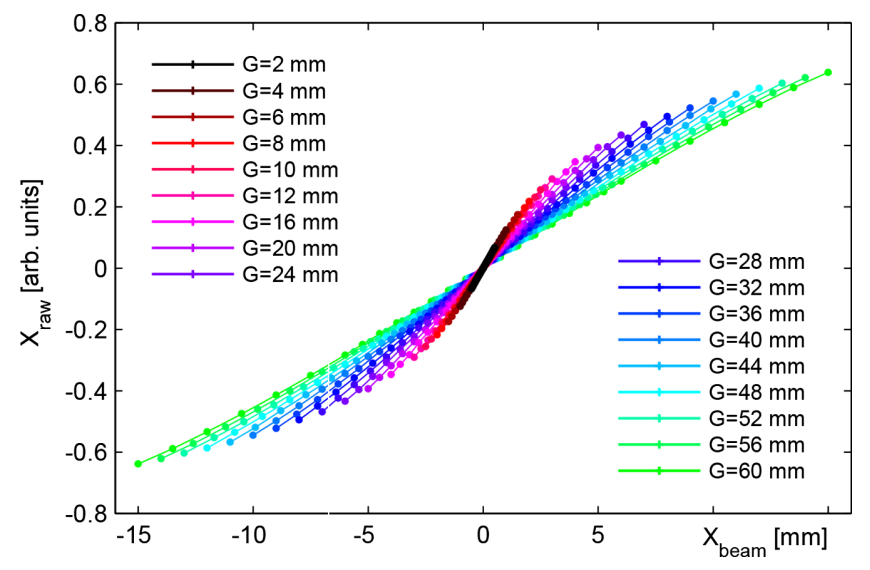

FIG. 6. Nonlinear relationship between $X_{\text {beam }}$ and $X_{\text {raw }}$, depending on the beam offset and jaw gap. 


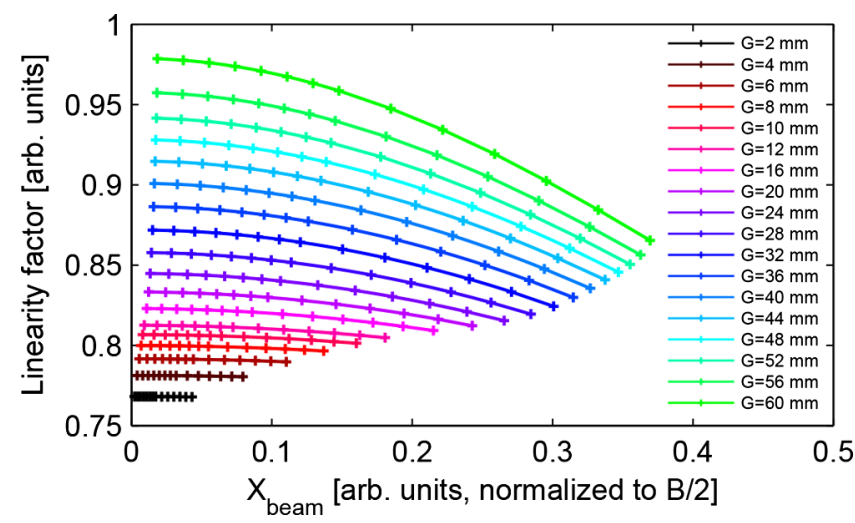

FIG. 7. Linearity factor as a function of the beam offset normalized to the BPM aperture.

in Fig. 7. They quantify the slope of geometrical nonlinearity of BPM readings with respect to button distance and beam position. It is convenient to plot $L_{f}$ against the beam offset $X_{\text {beam }}$ normalized by $B / 2$ so the aperture is always within $[-1,1]$ for all $G$. It can be seen that the $L_{f}$ curve is nonlinear for each set of beam positions at a corresponding jaw gap. The value of $L_{f}$ increases with aperture, however; even for the case of the largest gap, it changes by no more than $15 \%$ between the smallest and largest beam offset for the considered collimator.

The accuracy of the CST Wakefield solver depends directly on the mesh and calculation time step. Even with manual fixed mesh settings, the automatic hexahedral mesh is usually adapted by the CST mesher to the geometry at each sweep iteration, in this case depending on the jaw gap and the beam position. The maximum mesh count of the collimator model varied between $2.2 \times 10^{6}$ and $3 \times 10^{6}$ over iterative simulations of jaw gaps, while the sizes of the mesh cell edges were between 0.2 and $6 \mathrm{~mm}$. Each simulation took under 20 min on a moderate desktop PC (3 GHz Dual Core, 8 GB RAM).

The convergence of the Wakefield solver was also tested by reducing the mesh cell size of a fixed geometrical model. The solver had reached convergence within $\Delta V_{\text {peak }}=$ $2 \mathrm{mV}$ of peak voltage measured by the BPM. This can be translated to a position jitter of $20 \mu \mathrm{m}$ and defines the accuracy of considered simulations. Simulations of the BPM response for small beam offset steps comparable to $20 \mu \mathrm{m}$ are extremely sensitive and the results may be within noise level of the solver.

\section{B. Correcting BPM nonlinearities}

The behavior of collimator BPM signals for jaw gaps other than the ones simulated in Fig. 6 can be predicted through simulation and there is no need to simulate as many gaps as possible with small steps. Already based on the mapped values of simulated $X_{\text {raw }}$ on a grid of $X_{\text {beam }}$ for a set of jaw gaps, we have developed a fast and accurate algorithm which compensates the nonlinear BPM readings of feasible beam offsets within the full jaw motion range.

We can describe a relationship between the original beam positions $X_{\text {beam }}$, the aperture $B$, and the calculated raw positions $X_{\text {raw }}$ as an invertible function:

$$
X_{\text {raw }}=P\left(X_{\text {beam }}, B\right),
$$

so that when inverted, it would revert voltage readings back to position:

$$
X_{\text {beam }}=P^{-1}\left(X_{\text {raw }}, B\right)=Q\left(X_{\text {raw }}, B\right) .
$$

By fitting the surface $Q$ with a 2D $m n$-degree polynomial (see Fig. 8) with coefficients $c_{p q}$

$$
X_{\text {beam }} \approx X_{\text {bpm }}=\sum_{p, q=0}^{m, n} c_{p q} X_{\text {raw }}^{p} B^{q},
$$

one can use it to convert raw position data $X_{\text {raw }}$ at a given jaw gap back to the original beam position $X_{\text {beam }}$ with an accuracy mainly defined by absolute simulation accuracy and the polynomial's power. The resulting application of this polynomial is the approximate (corrected) beam position derived from raw BPM signals, so it can be denoted as $X_{\mathrm{bpm}}$. Because of smooth properties of the surface $Q$, the fitting scheme can be a general polynomial regression model in two dimensions. Already for $m=5$ and $n=3$ the rms error is 0.004 and goodness of fit $R^{2}=1$, so we can consider these powers as optimal for our requirements and refer to the polynomial as Poly53. Excluding negligibly small fit coefficients the Poly53 has the form of

$$
\begin{aligned}
X_{\mathrm{bpm}}= & c_{10} X_{\mathrm{raw}}+c_{11} X_{\mathrm{raw}} B+c_{30} X_{\mathrm{raw}}^{3}+c_{12} X_{\mathrm{raw}} B^{2} \\
& +c_{31} X_{\mathrm{raw}}^{3} B+c_{13} X_{\mathrm{raw}} B^{3}+c_{50} X_{\text {raw }}^{5}+c_{32} X_{\mathrm{raw}}^{3} B^{2},
\end{aligned}
$$

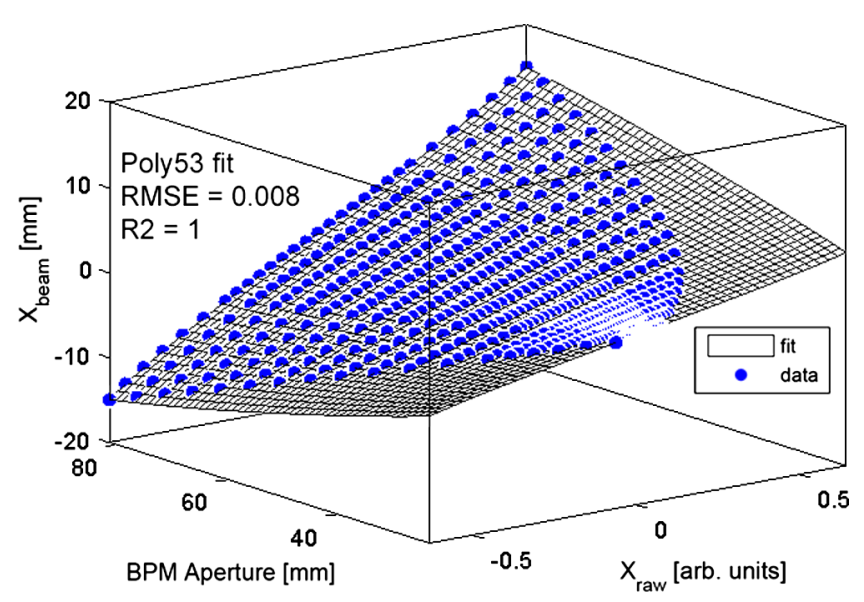

FIG. 8. The 2D polynomial fit (Poly53) used to correct the nonlinear BPM readings for full jaw motion range. 
TABLE II. Coefficients of the 2D surface fit, used to obtain the corrected beam position for full jaw motion range and maximum beam offset of $50 \% \mathrm{G}$.

\begin{tabular}{lc}
\hline \hline Coefficient & Value \\
\hline$c_{10}$ & -0.7179 \\
$c_{11}$ & 0.4047 \\
$c_{30}$ & -1.523 \\
$c_{12}$ & $-2.928 \mathrm{e}-3$ \\
$c_{31}$ & 0.06815 \\
$c_{13}$ & $2.47 \mathrm{e}-5$ \\
$c_{50}$ & 3.407 \\
$c_{32}$ & $1.897 \mathrm{e}-4$ \\
\hline
\end{tabular}

where $c_{p q}$ are the fit coefficients, listed in Table II. The coefficients of Poly53 are unique for a given collimator geometry and valid within jaw gaps and beam offsets within defined limits in the horizontal plane. The accuracy of beam position calculation by Poly53 within the BPM aperture $B$ is defined as the deviation of the corrected BPM reading from the original beam location:

$$
E_{x}=X_{\text {beam }}-X_{\text {bpm }}
$$

A corresponding error map is plotted in Fig. 9 as a function of the horizontal beam offset as a percentage of $G$ and the distance between BPM buttons $B$. Here, the colors represent values of $E_{x}$, which include the polynomial fitting error (up to $30 \mu \mathrm{m}$ ) and the numerical error of the wakefield solver (up to $20 \mu \mathrm{m}$ ). The overall correction error of Poly53 is well below $60 \mu \mathrm{m}$ for beams within $\pm G / 2$ for the full jaw motion range. It is clear that the drawback of the

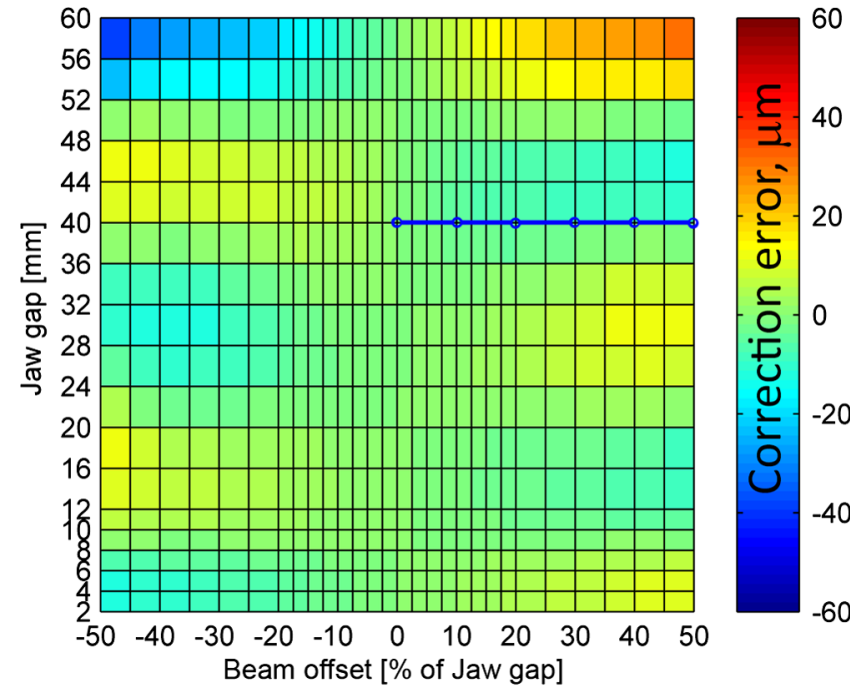

FIG. 9. An error map of BPM readings corrected with Poly53 for the full jaw motion range. The overall error inside considered limits is within $60 \mu \mathrm{m}$. The highlighted points are referenced by Fig. 11 and correspond to the position error within $\pm 10 \mu \mathrm{m}$. polynomial is its performance for largely displaced beams when $X_{\text {beam }} \rightarrow G / 2$, but in reality the beam is unlikely to have such a large offset.

\section{Nonlinearities of vertical offsets}

The collimator BPM readings cannot be used to identify a beam displacement in the orthogonal plane due to the fact that electrodes are positioned in the collimating plane. For an orthogonal beam offset, a gradual decrease of the left and right signals is expected. However, calibrating the BPM readout versus the orthogonal center of the collimator would require sweeping the beam on the orthogonal axis (looking into the observer in Fig. 1) with high resolution bumps, which is not feasible at the SPS.

In view of possible beam offsets in the orthogonal plane, Poly53 was tested on a simulated 2D grid of beam offsets with small vertical (up to $2 \mathrm{~mm}$ ) and large horizontal (up to $80 \%$ of $G$ ) displacements. Figures 10(a) and 10(b) compare the performance of a DOS linearization and of the Poly53 application to position calculation. Here $G=40 \mathrm{~mm}$ and $k_{x}=15.3$ for the DOS method. Figure 11 shows the correction errors of both methods for two rows of points, highlighted in Fig. 10. The Poly53 is more accurate than the DOS linearization by a factor 100 .

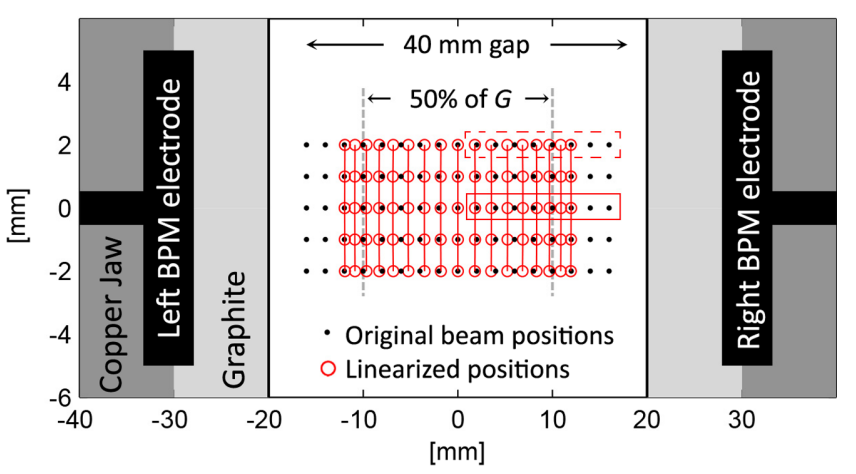

(a)

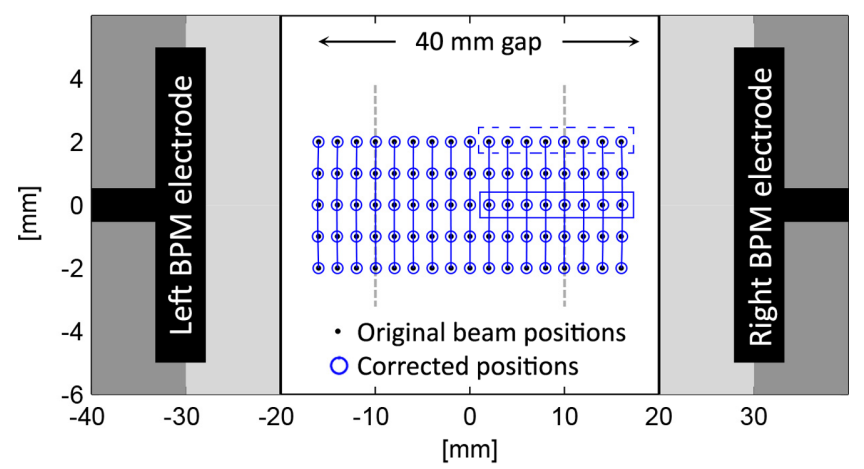

(b)

FIG. 10. Cross-section schematic of the collimator at BPM locations comparing performance of DOS linearization (top) and of Poly53 (bottom) correction methods for simulated beam sweeps in the transverse plane. 


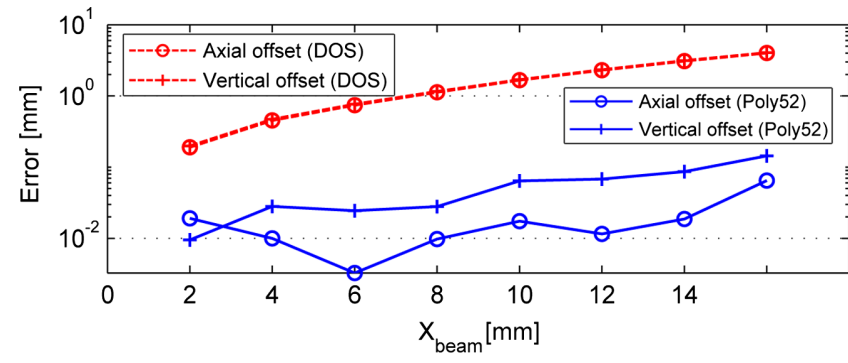

FIG. 11. Error of nonlinearity correction by DOS and Poly53 of horizontally offset points, and points with $\mathrm{X}$ and $\mathrm{Y}$ offsets. The considered points are highlighted correspondingly in Figs. 10(a) and 10(b). The points located on the $X$ axis and corrected with Poly53 are highlighted on the corresponding error map in Fig. 9.

\section{BPM electronics calibration}

The asymmetries between the cables and two electronics channels, which process signals from one pair of BPMs, introduce gains $g$ and offsets $o$ to the measured data [18]. The corrected electrode signal can be obtained as

$$
V_{j}^{\text {corr }}=g_{j} V_{j}+o_{j}
$$

where $j$ denotes the channel number corresponding to a particular jaw corner. The asymmetry errors are independent of the BPM aperture and beam offset. The parameters $g_{j}$ and $o_{j}$ (by default 1.0 and 0.0 , respectively) can be determined experimentally by swapping the opposite BPM channels and measuring the signals in these two configurations. The beam position is assumed to be constant during the test. If the beam orbit cannot be assumed, a second

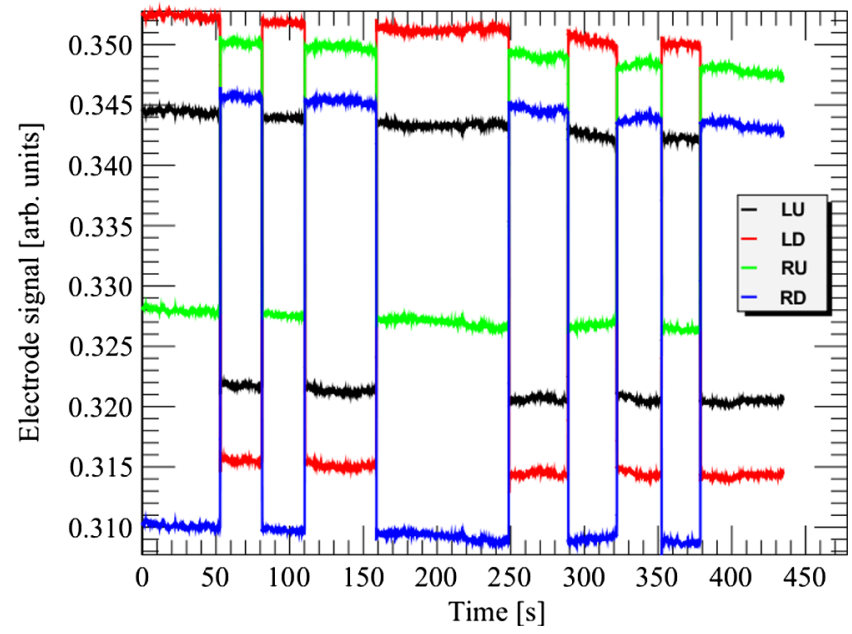

FIG. 12. BPM electrode signals and collimator jaw positions during the electronics calibration test. The BPM channels are flipped five times at a constant jaw gap of $30 \mathrm{~mm}$. The slight decrease in the signal amplitudes over time is due to the intensity decrease from normal beam losses.
TABLE III. Gain and offset coefficients applied to the received electrode data to counter for asymmetries in the acquisition electronics. The coefficients were verified to be very similar at different jaw gaps.

\begin{tabular}{ll}
\hline \hline Coefficient & Value \\
\hline$G_{\mathrm{LU}}$ & 1.0 \\
$G_{\mathrm{RU}}$ & 1.00508 \\
$G_{\mathrm{LD}}$ & 1.0 \\
$G_{\mathrm{RD}}$ & 0.97936 \\
$O_{\mathrm{LU}}$ & 0.0 \\
$O_{\mathrm{RU}}$ & 0.00409 \\
$O_{\mathrm{LD}}$ & 0.0 \\
$O_{\mathrm{RD}}$ & 0.00077 \\
\hline
\end{tabular}

calibration procedure with a common signal can be used, as described in [18].

Consider two channels 1 and 2, where $V_{11}$ and $V_{12}$ are signals from the electrodes 1 and 2 when they are connected to channels 1 and 2 , and $V_{21}$ and $V_{22}$ are the signals from the same electrodes when they are connected to channels 2 and 1 . Therefore if $g_{1}=1$, then

$$
g_{2}=\frac{V_{22}-V_{12}}{V_{11}-V_{21}} .
$$

The offset is determined in a similar manner, with $o_{1}=0$ and

$$
o_{2}=\frac{V_{11} V_{22}-V_{12} V_{21}}{V_{11}-V_{21}} .
$$

Five measurements were taken (see Fig. 12), and the values for each channel are listed in Table III. The standard deviation of the gains is $3 \times 10^{-4}$, while that for offsets is $7 \times 10^{-5}$.

\section{E. Measuring BPM nonlinearities}

An automated jaw scan was performed with the SPS prototype collimator to characterize the BPM readout for selected jaw gaps and beam offsets in order to validate the calibration and Poly53. At the start of the scan, the jaws were centered around the beam using feedback from BLMs with gain and offset calibration correction switched on. A horizontal jaw sweep was done by simultaneously moving the jaws around the circulating beam, keeping the jaws parallel at a fixed gap. The jaws were moved in steps of $\Delta J$, which include a subset of identical simulated beam steps of $\Delta X$ within $\pm G / 2$. The scan technique is similar to that performed in the initial feasibility studies [12], except that the automation allows a larger gap range and step resolution to be explored.

The scan was executed automatically by the same application software used to test the alignment procedure. The collimator jaws were first opened to parking positions $( \pm 30 \mathrm{~mm})$, and then were shifted by $0.5 \mathrm{~mm}$ every $3 \mathrm{~s}$. 


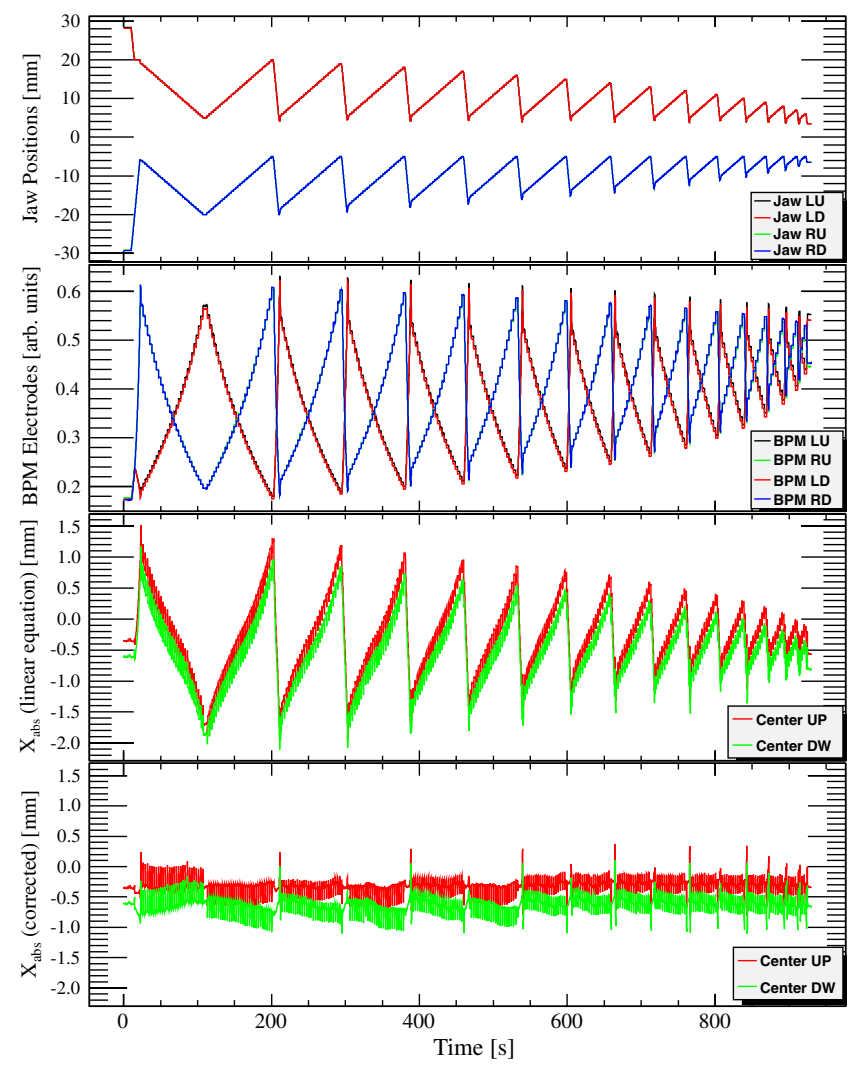

FIG. 13. Automatic collimator scan, where the jaw center is changed by $0.5 \mathrm{~mm}$ every $3 \mathrm{~s}$, and the jaw gap is reduced by $1 \mathrm{~mm}$ at the end of each iteration. The raw, linearized, and corrected by polynomial electrode signals are shown for comparison. The upstream and downstream jaw positions and electrode signals of each jaw are not distinguishable from each other due to a large vertical scale of both plots.

When one of the jaws reached a predefined limit set to avoid beam scraping, the jaws were moved back to the starting point and the jaw gap was reduced by $1 \mathrm{~mm}$. The procedure was repeated until a minimum jaw gap (typically $10 \mathrm{~mm}$ ) was reached. Sample results from a collimator scan are shown in Fig. 13. The measured beam axis $X_{\text {abs }}$ is shown with and without BPM nonlinearity correction by Poly53. The corrected beam axis data is constant except for periodic noise that occurs when the jaws are moved at each step, as well as from the end point back to the starting point of the scan. The jaw movement is nonlinear in time, but a linear interpolation is used to synchronize the $1 \mathrm{~Hz}$ collimator data to the $10 \mathrm{~Hz}$ BPM electrode data for plotting purposes.

To correlate the measured jaw scan results with simulations, it is necessary to translate the jaw sweep into beam sweep. If the jaws were positioned at locations

$$
J_{L, R, m}=J_{L, R}+m \times \Delta J
$$

where $m$ is an iterator over each scan point, we then can consider the original "moving" beam locations at

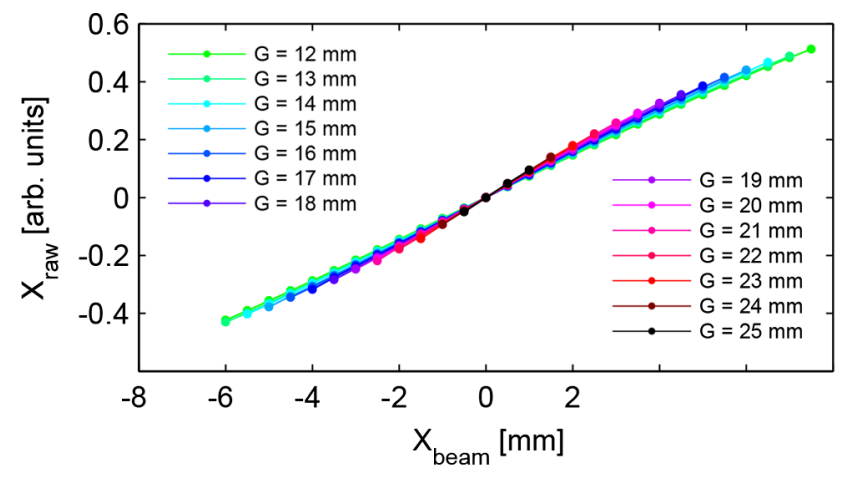

(a)

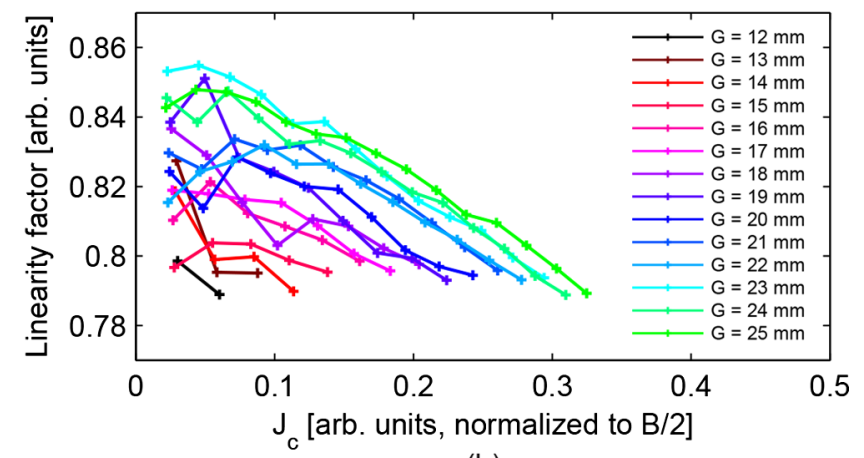

(b)

FIG. 14. Measurement results of the nonlinear relationship between the upstream BPM response and the beam offset.

$$
X_{\text {beam }, m}=J_{c, m}=\left(J_{R, m}+J_{L, m}\right) / 2
$$

and calculate the linearity factor as in Eq. (12).

There is a very good agreement between the simulated and measured BPM characteristics, shown in Figs. 6 and 14, respectively. The linearity factors of selected measured data are shown in Fig. 15 with comparison to simulated data (Fig. 7). The pattern of the measured $L_{f}$ is

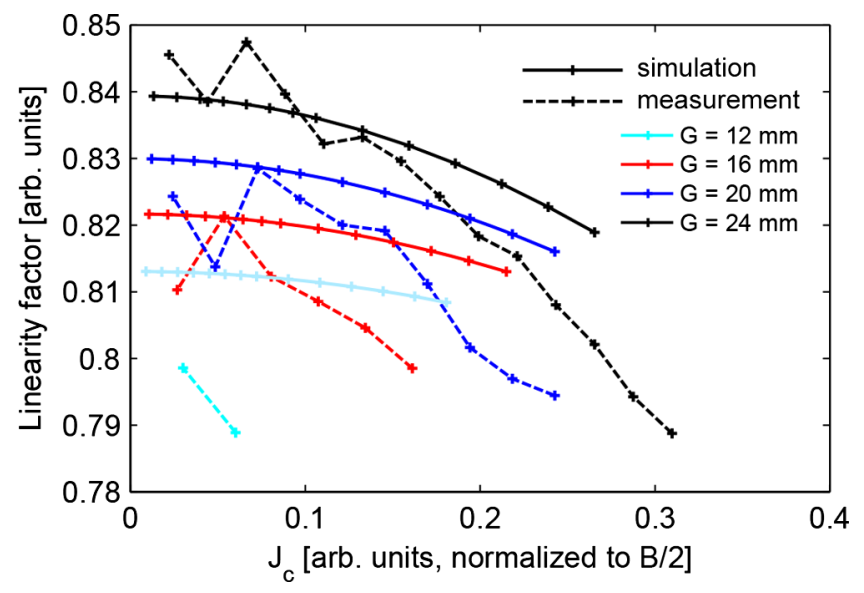

FIG. 15. Comparison of measured and simulated linearity factors of several BPM characteristics plots shown in Fig. 14. 
well visible, with a steeper slope towards large beam offsets. The noise in the measured $L_{f}$ is due to orbit drifts and beam intensity decrease, which results in a gradual attenuation of the electrode signals over time during the scan.

\section{BPM-BASED JAW ALIGNMENT}

\section{A. Algorithm}

The objective of the alignment is to minimize $X_{\mathrm{bpm}}$ in Eq. (6). A successive approximation algorithm was developed to automatically align the collimator jaws around the beam axis from any starting jaw gap and beam offset. A flowchart of the algorithm is shown in Fig. 16. The first step is to estimate what would be the aligned position for the jaw furthest from the beam, $J_{i}$ :

$$
J_{i}=2 X_{\mathrm{bpm}}+J_{i-1}
$$

where $i$ is an iterator over the successive approximation steps and $J_{i-1}$ is the current jaw position. Once the jaw reaches the supposedly aligned position, a new estimate is obtained for the beam axis using Eq. (11), and the above step is repeated. The loop has two terminating conditions. The first is when the measured beam axis is below the error which can be specified as an input parameter: $\left|X_{\mathrm{bpm}}\right| \leq X_{\text {error }}$. The second is when the jaw gap decreases below a minimum gap: $G \leq G_{\min }$. $G_{\min }$ can be calculated in terms of the beam size at the collimator to ensure that the jaws do not inadvertently scrape away the beam during the

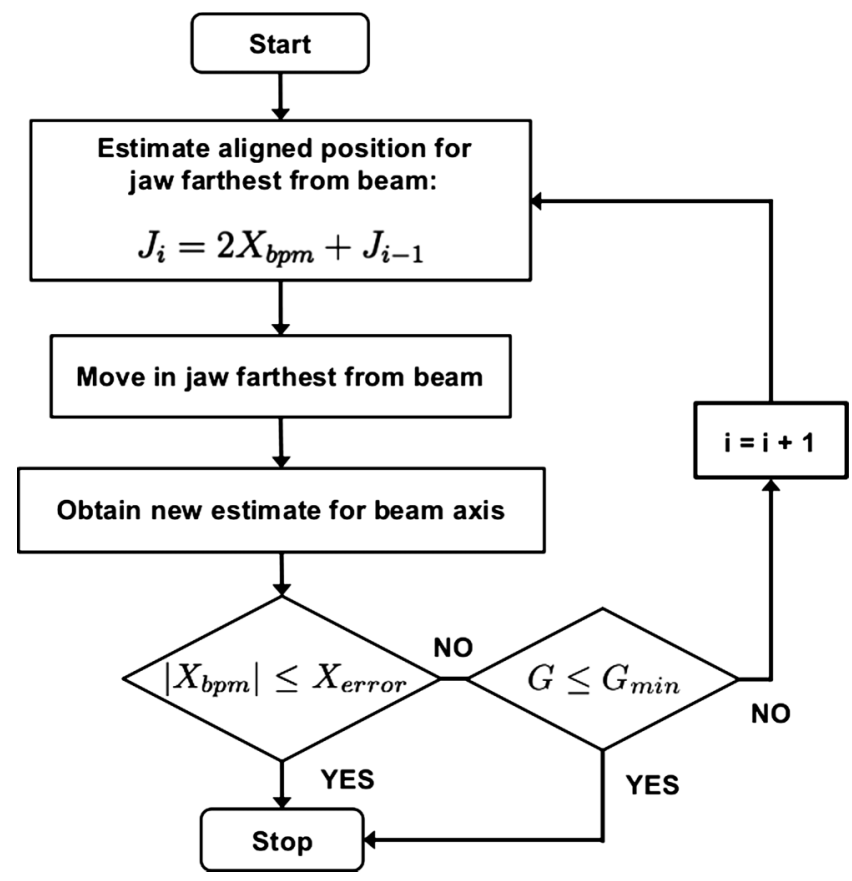

FIG. 16. Flowchart of the BPM-based successive approximation alignment algorithm. alignment procedure, with a margin large enough to cater for any optics errors which might affect the beam size. If $G=G_{\min }$, the algorithm attempts to continue the alignment by moving the jaw closest to the beam outwards, rather than the jaw farthest from the beam inwards.

The alignment algorithm was implemented in the existing Java application used to control the prototype collimator in SPS [21]. The algorithm implementation is flexible and allows the use of both the linearized beam axis equation and the polynomial correction. In addition, the gains and offsets for the electronics, as well as the BPM nonlinearity polynomial coefficients, can be modified on the fly if necessary.

\section{B. Alignment tests}

A single LHC-type bunch circulating in the SPS with an injection intensity of $1.2 \times 10^{11} \mathrm{p}$ was used to test the automatic collimator alignment algorithm at an energy of $270 \mathrm{GeV}$. A total of 19 alignment tests were conducted in two fills, and the alignment parameters were changed for each test, as listed in Table IV. Two types of starting jaw positions were considered. In the first case, the jaws were initially set at parking positions around the absolute axis (zero position). In the second case, the jaws were initially positioned off center, with $\left|J_{c}\right|>3.5 \mathrm{~mm}$.

The alignment time was strongly influenced by the time interval between each step, the alignment accuracy required, and, to a lesser degree, by the initial jaw gap as the BPM nonlinearities are proportional to the gap. The collimator jaw corner positions, raw electrode signals, and measured beam axis during a typical automatic alignment are shown in Fig. 17. Approximately 11 and 4 steps were required with the left and right jaws, respectively, until they are finally aligned after $30 \mathrm{~s}$, when the electrode signals in the first plot are equalized. The shortest alignment time achieved was $\sim 20 \mathrm{~s}$, a factor 6 improvement over the best achieved alignment time of $\sim 120 \mathrm{~s}$ with the BLM-based technique.

\section{Comparison with BLM-based alignment}

For each new fill, the jaws were first aligned using feedback from the BLMs. This involves moving each jaw in steps of 50 to $100 \mu \mathrm{m}$ until a loss spike is observed in the closest BLM downstream of the collimator. A comparison of the beam axis measured with the BPM-based and BLMbased techniques is shown in Fig. 18. The averages of the upstream and downstream positions are plotted for the BPM-based alignments, as the jaw corners are not aligned individually in the BLM-based alignments. The centers are in agreement within less than $150 \mu \mathrm{m}$ in most instances, although larger deviations are observed in the second fill possibly due to orbit drifts. It is important to note that the design positioning tolerance of the BPM buttons with respect to the jaw surface is $50 \mu \mathrm{m}$. However, systematic calibration with the BLM-based alignment in the LHC 
TABLE IV. Alignment parameters and the corresponding alignment times achieved for a subset of the tests.

\begin{tabular}{lcccccccccc}
\hline \hline & \multicolumn{10}{c}{ Alignment trials } \\
\cline { 2 - 11 } Parameter & 1 & 2 & 3 & 4 & 5 & 6 & 7 & 8 & 9 & 10 \\
\hline Step interval $[\mathrm{s}]$ & 5 & 2 & 2 & 5 & 1 & 2 & 1 & 1 & 1 & 1 \\
Accuracy $[\mu \mathrm{m}]$ & 5 & 1 & 5 & 5 & 5 & 1 & 10 & 5 & 1 & 1 \\
Initial jaw gap [mm] & 50 & 60 & 60 & 48 & 35 & 35 & 35 & 21 & 21 & 35.5 \\
Initial jaw center [mm] & 0.00 & 0.00 & 0.00 & 6.00 & 7.50 & 7.50 & -7.50 & -3.50 & 4.50 & 12.25 \\
Final jaw gap [mm] & 35.93 & 43.15 & 58.50 & 35.37 & 19.93 & 19.65 & 20.45 & 13.90 & 11.39 & 10.44 \\
Alignment time [s] & 47 & 105 & 29 & 81 & 17 & 52 & 23 & 26 & 24 & 34 \\
\hline
\end{tabular}

should reduce this inaccuracy to $10 \mu \mathrm{m}$. This, combined with the inaccuracies introduced by the large jaw step size required due to the larger beam sizes in the SPS, provides a valid explanation for the differences in the beam center measurements between BLM-based and BPM-based alignment.

\section{Effects of beam offsets in the orthogonal plane}

The BPMs are positioned in the SPS prototype collimator to measure the beam offset in the horizontal plane.

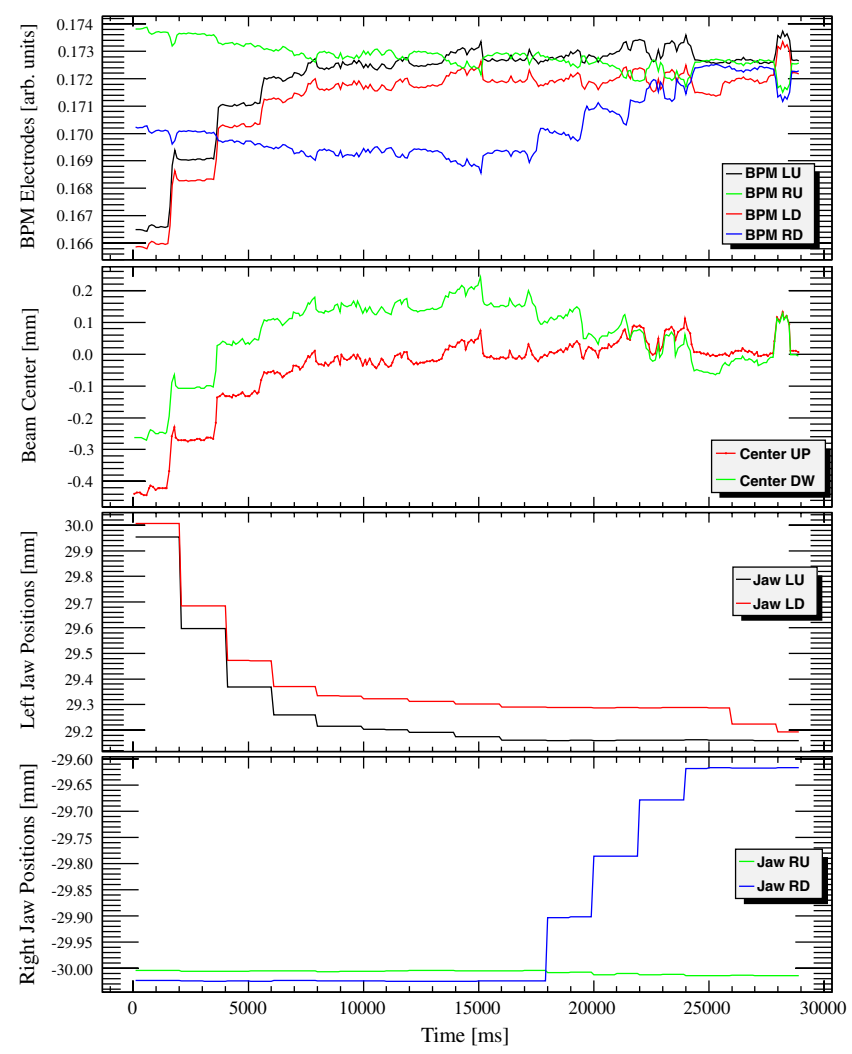

FIG. 17. Collimator jaw corner positions, raw electrode signals, and measured position of the beam $X_{\text {bpm }}$ during a typical automatic alignment in the SPS. The polynomial nonlinearity correction of BPMs is not used to ensure that no additional errors are introduced.
Vertical beam offsets could affect the measurements [see Eq. (5)]. Hence, a test was conducted to verify the extent of the measurement errors for vertical bumps. The first step was to position the jaws symmetrically around the beam using the automatic BPM-based alignment algorithm. Then, vertical orbit bumps of $+2.5 \mathrm{~mm}$ followed by $-2.5 \mathrm{~mm}$ were introduced at the collimator location.

The change in the measured BPM electrodes and the corresponding shift in the measured beam axis (taking into account a jaw gap of $20 \mathrm{~mm}$ ) are shown in Fig. 19. A minor shift of $\sim 50 \mu \mathrm{m}$ was detected, which is not negligible for LHC operational purposes considering the magnitude of the orbit bump introduced. The effect in the downstream corner BPM electrode signals is more evident for the negative orbit bump, and vice versa for the positive orbit bump, as the bump cannot be applied at the same longitudinal position in both cases. As concluded in Sec. IV C, the beam position calculation and suppression of the nonlinearity effect in the orthogonal plane can be handled by the correction polynomial Poly53 without any problem.

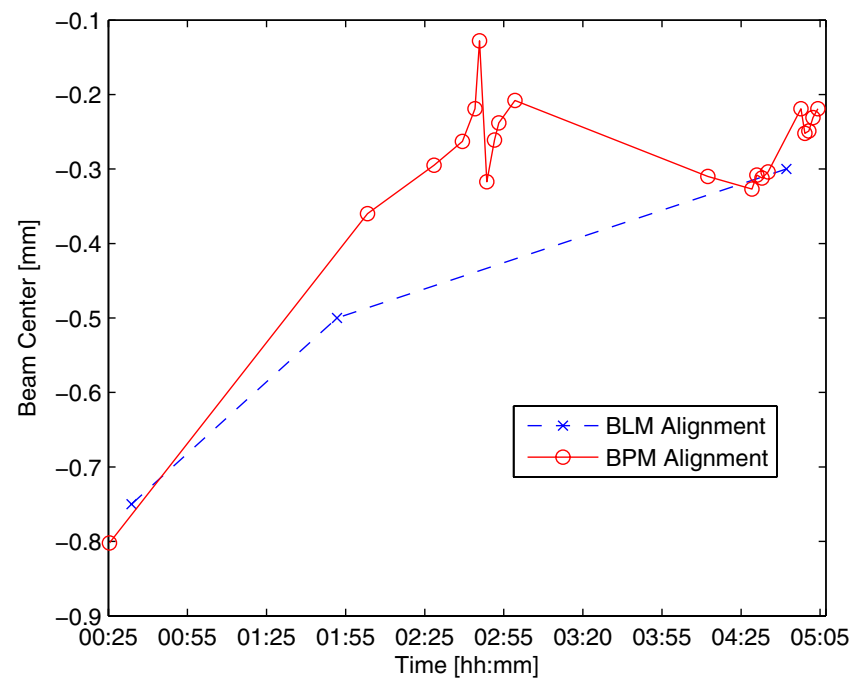

FIG. 18. Comparison of the beam axis measured with the BLM-based and BPM-based alignment techniques. The SPS was refilled twice at $00: 20$ and $01: 34$. 


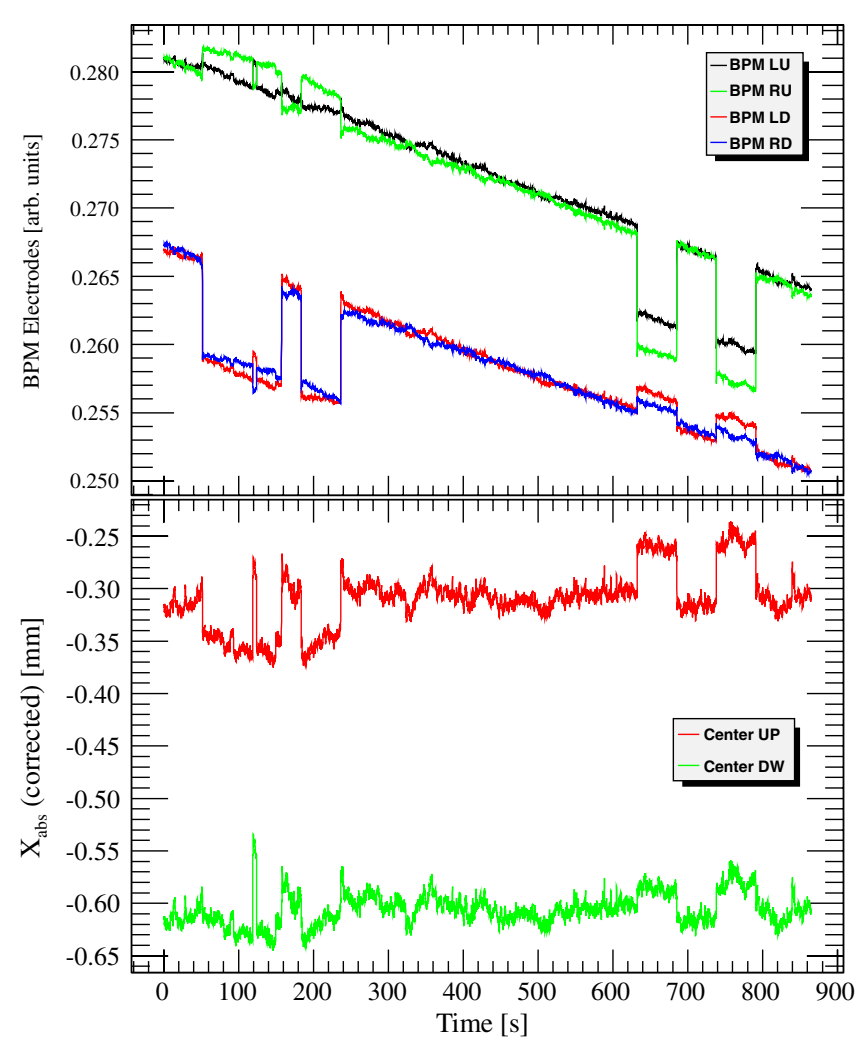

FIG. 19. Changes in the measured BPM signals and the calculated beam axis due to vertical orbit bumps of $+2.5 \mathrm{~mm}(100-300 \mathrm{~s}, \mathrm{ON}-\mathrm{OFF}-\mathrm{ON})$ and $-2.5 \mathrm{~mm}(650-850 \mathrm{~s}$, ON-OFF-ON).

\section{SUMMARY AND OUTLOOK}

Collimators with embedded BPMs will be installed in the LHC to monitor the beam position at the collimator locations with greater precision. An algorithm for automatically centering the jaws around the beam, based on feedback from the BPM readouts, was described. The nonlinear effects of beam position measurements are corrected by an efficient $2 \mathrm{D}$ polynomial in the whole jaw motion range well within $50 \mu \mathrm{m}$ accuracy. Results from tests with a prototype collimator in the SPS were presented, where an alignment time of $\sim 20 \mathrm{~s}$ was reached with a precision between opposite electrode signals corresponding to $5 \mu \mathrm{m}$, at jaw gaps as large as $58 \mathrm{~mm}$.

The algorithm will be used for collimator alignment in the LHC starting from 2015, where the BPMs could allow for fill-to-fill alignments and also be integrated into the LHC beam orbit feedback system. This would decrease the necessary TCT alignment time from an hour to only seconds, which, if compared to alignments held during the 2012 LHC run, is an improvement of 2 orders of magnitude. The BPMs can also allow for online orbit monitoring and provide the possibility of placing interlocks on the beam orbit, which would dump the beam if abnormally large orbit shifts are detected. However, any collimator movements will have to be studied in detail to ensure that no additional risks are introduced for machine protection.

\section{ACKNOWLEDGMENTS}

The authors would like to thank S. Cettour Cave, J. Dalla-Costa, S. Massot, A. Moscatelli, D. Perez, and E. Veyrunes, the SPS operators on shift during the MDs, as well as G. Rumolo, the SPS beam test coordinator. Discussions with V. Baggiolini regarding the UDP packet transmission implementation for the tests were also appreciated.

[1] CERN, Report No. CERN-2004-003-V1, edited by O. S. Brüning, P. Collier, P. Lebrun, S. Myers, R. Ostojic, J. Poole, and P. Proudlock, 2004.

[2] R. W. Aßmann et al., in Proceedings of the 8th European Particle Accelerator Conference, Paris, France, 2002, (EPS-IGA and CERN, Geneva, 2002), pp. 197-199.

[3] R. W. Aßmann. et al., in Proceedings of the 9th European Particle Accelerator Conference, Lucerne, Switzerland, 2004 (EPS-AG, Lucerne, 2004), pp. 536-538.

[4] R.W. Aßmann et al., in Proceedings of the 10th European Particle Accelerator Conference, Edinburgh, Scotland, 2006 (EPS-AG, Edinburgh, Scotland, 2006), pp. 1538-1540.

[5] R. W. Aßmann, E. Holzer, J.-B. Jeanneret, V. Kain, S. Redaelli, G. Robert-Demolaize, and J. Wenninger, in Proceedings of the 9th European Particle Accelerator Conference, Lucerne, Switzerland (EPS-AG, Lucerne, 2004), pp. 1825-1827.

[6] D. Wollmann et al., in Proceedings of the International Particle Accelerator Conference, IPAC'10, Kyoto, Japan, 2010 (ICR, Kyoto, 2010), pp. 1237-1239.

[7] G. Valentino, R. W. Aßmann, R. Bruce, S. Redaelli, A. Rossi, N. Sammut, and D. Wollmann, Phys. Rev. ST Accel. Beams 15, 051002 (2012).

[8] G. Valentino, R. W. Aßmann, R. Bruce, S. Redaelli, and N. Sammut, in Proceedings of the IEEE UKSim-AMSS 6th European Modeling Symposium on Mathematical Modeling and Computer Simulation, Valletta, Malta, 2012 (IEEE, New York, 2012).

[9] G. Valentino, R. W. Aßmann, R. Bruce, and N. Sammut, in Proceedings of the IEEE 10th International Symposium on Applied Machine Intelligence and Informatics, Herl'any, Slovakia, 2012 (IEEE, New York, 2012), pp. 355-358.

[10] A. Dallocchio, A. Bertarelli, C. Boccard, F. Carra, M. Gasior, L. Gentini, and M. Timmins, in Proceedings of the 2nd International Particle Accelerator Conference, IPAC'11, San Sebastián, Spain, 2011 (EPS-AG, Spain, 2011), pp. 1611-1613.

[11] R. Bruce, R. W. Aßmann, L. Lari, and S. Redaelli, in Proceedings of the Machine Protection Workshop, Annecy, France, 2013 (CERN, Geneva, 2013).

[12] D. Wollmann, O. Aberle, R. W. Assmann, A. Bertarelli, C. Boccard, R. Bruce, F. Burkart, M. Cauchi, A. 
Dallocchio, D. Deboy, M. Gasior, R. Jones, S. Redaelli, A. Rossi, and G. Valentino, in Proceedings of the 2nd International Particle Accelerator Conference, IPAC'11, San Sebastián, Spain, 2011 (EPS-AG, Spain, 2011), pp. 3747-3749.

[13] S. Redaelli, R. W. Aßmann, R. Bruce, A. Rossi, and D. Wollmann, in Proceedings of the 46th ICFA Advanced Beam Dynamics Workshop (Jacow, CERN, Geneva, 2010), pp. 395-399.

[14] A. Bertarelli, C. Boccard, A. Dallocchio, M. Gasior, L. Gentini, and A. Nosych, in Proceedings of the 10th European Workshop on Beam Diagnostics and Instrumentation of Particle Accelerators (Jacow, CERN, Geneva, 2011), pp. 80-82.

[15] P. Piot, Fermilab Report No. Beams-doc-1894-v1, 2005.

[16] M. Gasior, J. Olexa, and R. J. Steinhagen, in Proceedings of the Beam Instrumentation Workshop (Jacow, CERN, Geneva, 2012).
[17] M. Gasior, J. Olexa, and R. J. Steinhagen, in Proceedings of the 10th European Workshop on Beam Diagnostics and Instrumentation of Particle Accelerators (Jacow, CERN, Geneva, 2011), pp. 98-100.

[18] M. Gasior, in Proceedings of the International Beam Instrumentation Conference (Jacow, CERN, Geneva, 2013).

[19] CST Particle Studio, http://www.cst.com/Content/Products/ PS/Overview.aspx.

[20] D. Wollmann, O. Aberle, R. W. Assmann, A. Bertarelli, C. Boccard, R. Bruce, F. Burkart, M. Cauchi, A. Dallocchio, D. Deboy, M. Gasior, R. Jones, V. Kain, L. Lari, A. Nosych, S. Redaelli, A. Rossi, and G. Valentino, in Proceedings of the 52nd ICFA Advanced Beam Dynamics Workshop (Jacow, CERN, Geneva, 2012).

[21] S. Redaelli, R. W. Aßmann, M. Jonker, and M. Lamont, CERN-EDMS Report No. LHC-TCT-ES-0001, 2007. 